Arq. Bras. Med. Vet. Zootec., v.68, n.5, p.1283-1291, 2016

\title{
Influência da condição corporal ao parto no balanço energético e desempenho reprodutivo de cabras leiteiras no pós-parto
}

\author{
[Influence of body condition at calving on energy balance and reproductive performance \\ of dairy goats in the postpartum] \\ L.P. Barbosa ${ }^{1}$, M.T. Rodrigues ${ }^{2}$, J.D. Guimarães ${ }^{2}$, C.A.A. Torres ${ }^{2}$, G.R. Carvalho ${ }^{2}$, \\ L.S. Amorim ${ }^{2}$, P.A. Dutra ${ }^{3}$ \\ ${ }^{1}$ Universidade Federal do Recôncavo da Bahia - Cruz das Almas, BA \\ ${ }^{2}$ Universidade Federal de Viçosa - Viçosa, MG \\ ${ }^{3}$ Universidade Federal da Bahia - Salvador, BA
}

\begin{abstract}
RESUMO
O estudo teve como objetivo avaliar a influência da condição corporal $(\mathrm{CC})$ ao parto no balanço energético (BE) e o desempenho reprodutivo de cabras Alpinas no pós-parto. Foram utilizadas 68 cabras distribuídas em três grupos (G), sendo: G1- cabras com baixa CC (CC entre 1,5 e 2,5); G2: cabras com moderada CC (entre 2,75 e 3,5) e G3: cabras com alta CC (entre 3,75 e 5,0). Os animais receberam dieta (silagem de milho e concentrado, com $18 \%$ de proteína bruta e $1,7 \mathrm{Mcal} / \mathrm{kg}$ de matéria seca de energia líquida) ad libitum. As avaliações foram feitas nas oito primeiras semanas de lactação, para determinação do $\mathrm{BE}$, do intervalo do parto à primeira ovulação e ao primeiro estro e da concentração plasmática de progesterona. Os dados foram analisados por análise de variância a $5 \%$ de probabilidade. Não houve efeito da $\mathrm{CC}$ ao parto sobre o $\mathrm{BE}$ dos animais. Todas as cabras apresentaram BE negativo ao parto, com mudança para BE positivo aos 51, 58 e 64 dias pós-parto, respectivamente. Não houve diferença na concentração plasmática de progesterona nos três grupos, sendo caracterizada função luteal (concentração $\geq 1 \mathrm{ng} / \mathrm{dL}$ ) em apenas $5,9 \%$ dos animais avaliados. $\mathrm{O}$ intervalo do parto à primeira ovulação foi de 46 dias. Apenas 4,4\% das cabras exibiram estro. Cabras leiteiras de média produção, entre 2,5 e 3,0kg de leite/dia, entram em BE negativo ao parto, independentemente da CC apresentada, influenciando negativamente o reinício da atividade ovariana no pós-parto.
\end{abstract}

Palavras-chave: caprinos, ciclicidade pós-parto, progesterona

\begin{abstract}
The concentration of progesterone in the blood was used to study the effect of body condition score (BCS) on the post-partum ovarian activity of dairy goats. Sixty-eight female goats were distributed between three treatments. Treatment 1: goats with BCS between 1.00 and 2.75; Treatment 2: goats with BCS between 2.75 and 3.50; and Treatment 3: goats with BCS between 3.50 and 5.00. The goats had the blood collected immediately after birth, and from that time up to the eighth week post-partum at three day intervals. The progesterone analysis on the blood was used to determine the interval between the birth and the first ovulation. The females were teasing two times per day for estrus detection and determination of the time interval from birth to first estrus. No significant difference was found on the progesterone concentration between treatments. The luteal functionality was characterized in only $5.9 \%$ of goats, with a concentration higher than $1 \mathrm{ng} / \mathrm{mL}$. The period from birth to first ovulation was 46 days. The detectable estrus was found in only $4.4 \%$ of goats. The BCS at birth did not influence the recovery of ovarian activity of dairy goats.
\end{abstract}

Keywords: goats, post-partum cyclicity, progesterone

Recebido em 14 de abril de 2015

Aceito em 18 de fevereiro de 2016

E-mail: larissa@ufrb.edu.br 


\section{INTRODUÇÃO}

A eficiência reprodutiva é um dos principais fatores a serem considerados para aumentar a eficiência produtiva e a econômica em sistemas de produção, podendo ser maximizada pelo decréscimo do intervalo entre o parto e a primeira concepção (Hamadeh et al., 2001). Contudo, a duração desse intervalo está diretamente relacionada com as deficiências hormonais, de energia e as mudanças metabólicas que ocorrem no período pós-parto.

A perda de peso corporal neste período e o estabelecimento do balanço energético negativo podem influenciar negativamente o retorno ao estro e à atividade ovariana (Mbayahaga et al., 1998). No que tange ao efeito da perda de peso corporal e, consequentemente, de escore corporal no reinício da atividade ovariana no pós-parto em ovelhas, Gonzales et al. (1987) relataram que fêmeas que apresentam grandes perdas de peso tiveram um período de anestro pós-parto maior, maiores falhas do primeiro estro, falhas na primeira ovulação e redução do diâmetro médio dos folículos, bem como diminuição da frequência de picos de hormônio luteinizante (LH).

Considerando-se que a avaliação da condição corporal (CC) pode indicar estratégias de manejo nutricional e reprodutivo no período após o parto, este estudo teve como objetivo avaliar o efeito da $\mathrm{CC}$ ao parto no balanço energético (BE) e no desempenho reprodutivo de cabras leiteiras no pós-parto.

\section{MATERIAL E MÉTODOS}

O presente estudo foi realizado no Setor de Caprinocultura do Departamento de Zootecnia da Universidade Federal de Viçosa, no município de Viçosa/MG. Foram utilizadas 68 cabras da raça Alpina, selecionadas quanto à condição corporal (CC), à ordem de parto (segunda e terceira parições), à produção leiteira (2,5 a 3,0kg/dia) e sem alterações clínicas.

Os animais foram confinados em baias individuais de piso ripado, com dimensão de 1,5 $\mathrm{x} 2,0 \mathrm{~m}$. Todas as cabras receberam a mesma dieta, composta por silagem de milho, farelo de soja, fubá de milho, calcário calcítico e sal mineralizado (Tab. 1), com $18 \%$ de PB e
1,7Mcal/kg de MS de energia líquida (EL) (The Nutrition..., 1997), na forma de mistura completa, sendo fornecida duas vezes ao dia (às 7 h30 e às 17 horas), com 10\% de sobra e água ad libitum.

Tabela 1. Ingredientes utilizados (\% MS) e composição bromatológica da ração

\begin{tabular}{|c|c|}
\hline Ingrediente & $\%$ MS da dieta \\
\hline Silagem de milho & 52,94 \\
\hline Fubá de milho & 27,25 \\
\hline Farelo de trigo & 11,03 \\
\hline $\begin{array}{l}\text { Farelo de glúten de } \\
\text { milho }\end{array}$ & 5,00 \\
\hline Ureia & 1,20 \\
\hline Minerais & 2,58 \\
\hline & $\begin{array}{l}\text { Composição } \\
\text { bromatológica }\end{array}$ \\
\hline $\mathrm{PB}(\%)$ & 18,00 \\
\hline $\operatorname{PDR}(\%)$ & 11,50 \\
\hline PNDR $(\%)$ & 6,85 \\
\hline FDN (\%) & 31,46 \\
\hline FDA $(\%)$ & 17,57 \\
\hline $\mathrm{EE}(\%)$ & 2,60 \\
\hline CNE $(\%)$ & 38,00 \\
\hline Lignina $(\%)$ & 1,27 \\
\hline Cinzas $(\%)$ & 1,18 \\
\hline $\mathrm{Ca}(\%)$ & 0,80 \\
\hline $\mathrm{P}(\%)$ & 0,30 \\
\hline EL (Mcal/kg de MS $)^{1}$ & 1,70 \\
\hline
\end{tabular}

Os animais foram distribuídos em três grupos (G), com base na avaliação da CC ao parto, segundo Morand-Fehr e Hervieur (1999), em uma escala de 1 a 5 , com intervalos de 0,25 ponto, sendo G1: cabras com baixa CC (1,5 a 2,5) $(\mathrm{n}=26), \mathrm{G} 2$ : cabras com moderada $\mathrm{CC}(2,75$ a 3,5) $(\mathrm{n}=27)$ e $\mathrm{G} 3$ : cabras com alta $\mathrm{CC}(3,75$ a $5,0)(n=15)$.

Os parâmetros avaliados foram $\mathrm{BE}$, intervalo do parto à primeira ovulação, intervalo do parto ao primeiro estro e concentração plasmática de progesterona. O período de avaliações e coletas de amostras foi compreendido entre o parto e a oitava semana pós-parto.

Para determinação do balanço energético dos animais, a produção leiteira foi determinada diariamente e individualmente. Amostras semanais de leite foram coletadas de cada animal pela manhã e à tarde, para avaliação dos 
constituintes do leite (gordura, proteína, lactose e extrato seco total), por espectrofotometria de infravermelho. Os resultados de produção leiteira foram corrigidos para 3,5\% de gordura, por meio da fórmula demonstrada por Adams et al. (1995).

PLC $3,5 \%=(0,4255 \times$ PL $)+[16,425 \times(\% \mathrm{G} \div$ $100) \times$ PL], em que: PLC $3,5 \%=$ produção de leite corrigida para $3,5 \%$ de gordura; $\mathrm{PL}=$ produção de leite (g/dia); \%G = teor de gordura do leite.

Realizou-se pesagem diária da dieta fornecida aos animais e das sobras coletadas no dia seguinte, para posterior avaliação do consumo diário individual. Coleta de amostras das sobras de cada animal foi feita três vezes por semana, para análise bromatológica. Procedeu-se à pesagem semanal dos animais, antes do fornecimento da dieta da manhã, sendo a primeira pesagem realizada imediatamente após o parto.

O consumo de energia foi estimado com base no cálculo do NDT segundo Van Soest (1994), transformado em energia líquida, segundo Moe e Tyrell (1975). Para cálculo do balanço energético, foi utilizada a fórmula descrita a seguir: BE $(\mathrm{kcal})=$ consumo diário $\mathrm{EL}$ - EL mantença - EL lactação - EL ganho peso + EL perda peso, em que: EL mantença $(\mathrm{kcal})=$ $\left(\mathrm{kg}^{0,75}\right) \times 0,08 \mathrm{kcal} / \mathrm{kg}^{0,75}$, em que: 0,08 referese à exigência de mantença e EL lactação (kcal) $=$ produção leite diária $\times(0,312+(0,0962 \times \%$ gordura do leite).

Para determinação do intervalo do parto à primeira ovulação, os animais foram submetidos à coleta de sangue na veia jugular, para análise da concentração plasmática de progesterona. A primeira coleta foi realizada imediatamente após o parto, seguida de coletas com intervalo de 3,5 dias (duas vezes por semana), até o final das oito semanas. O sangue foi coletado em tubos a vácuo contendo anticoagulante EDTA. Imediatamente após a coleta, as amostras foram submetidas à centrifugação, durante 15 minutos a $336,3 \mathrm{G}$, e armazenadas a $-20^{\circ} \mathrm{C}$ em microtubos com capacidade de $1,5 \mathrm{~mL}$. A concentração plasmática de progesterona foi determinada por meio de radioimunoensaio (RIA), utilizando-se kits comerciais (DPC Medlab ${ }^{\circledR}$ ), em fase sólida.
Para determinação do intervalo do parto ao primeiro estro, foi realizada detecção de estro com o auxílio de rufião, duas vezes ao dia (às 10 e às 17 horas), a partir da primeira semana do período pós-parto.

Para análise dos dados, utilizou-se análise de variância a $5 \%$ de probabilidade. Para a variável balanço energético, foi utilizado o procedimento Linear Response Plateau, para ajustar modelos de regressão descontinua.

\section{RESULTADOS E DISCUSSÃO}

Não houve efeito da $\mathrm{CC}$ ao parto sobre o BE pósparto das fêmeas $(\mathrm{P}>0,05)$. Os animais, independentemente da $\mathrm{CC}$, encontravam-se em balanço energético negativo (BEN) ao parto, havendo uma recuperação gradativa nas semanas subsequentes, com mudança para BE positivo aos 51, 58 e 64 dias pós-parto para cabras com baixa $\mathrm{CC}, \mathrm{CC}$ moderada e com alta $\mathrm{CC}$, respectivamente (Fig. 1).

O início da lactação está associado invariavelmente a um período de BEN, em que o consumo de energia líquida fica aquém da demanda de energia para a produção de leite. Estudos consistentes que visem ao esclarecimento dessa informação são escassos, e ainda há falta de informações sobre qual a melhor CC ao parto. Nesse sentido, tais estudos poderiam ser importantes para se delinear dietas para programas de alimentação diferenciados em cabras leiteiras. Assim, Barbosa et al. (2009) observaram que o consumo de matéria seca (CMS), de fibra em detergente neutro (CFDN), de proteína bruta (CPB) e de energia líquida (CEL) não foi alterado em função da $\mathrm{CC}$ ao parto quando dietas de alta qualidade e alto nível de energia foram ofertadas a cabras leiteiras no pósparto.

A CC é um dos principais fatores que contribuem para a diminuição do consumo alimentar no pósparto (Parr et al., 1993). Fêmeas com CC elevada exibem maior depressão no apetite, desenvolvendo balanço energético negativo mais pronunciado, o que pode ocasionar maior mobilização das reservas corporais e maior acúmulo de triglicerídeos no fígado (Rukkwamsuk et al., 1999). Lago et al. (2001) observaram, em vacas leiteiras com maior $\mathrm{CC}$ ao parto, uma acentuada redução no peso e na $\mathrm{CC}$ 
durante o período de lactação em relação àquelas com CC baixa. Estes dados reforçam a ideia de que animais com mais reservas adiposas ao parto tendem a mobilizar essas reservas de forma acentuada (Pedron et al., 1993), havendo, portanto, uma relação direta entre perda de peso e mobilização de lipídeos (Vazquez-Anon et al., 1994).

Entretanto, para as condições do presente experimento, com cabras de média produção de leite, não foi observado esse efeito independente da CC ao parto. Em particular, após o parto, os baixos níveis de glicose e de insulina, associados a altas concentrações de hormônios de crescimento e ao estresse às catecolaminas, regulam as enzimas lipogênicas e lipolíticas no tecido adiposo (Vernon, 1981). As concentrações plasmáticas de ácidos graxos não esterificados (AGNE) estão positivamente correlacionadas ao grau de deficiência de energia e podem potencialmente enviar um sinal referente à condição nutricional aos centros neurais (Walters et al., 2002). Neste sentido, observou-se que, em cabras leiteiras de diferentes $\mathrm{CC}$ ao parto, as concentrações de AGNE parecem não ser influenciadas pelas semanas de lactação, conforme observado por Barbosa et al. (2009) e Rodrigues et al. (2006). E, ainda, o fornecimento de uma dieta de alta qualidade e com alta concentração de energia a cabras de média produção de leite aponta para a minimização de alterações do metabolismo energético dessa fase de pós-parto.

Nas mesmas condições do presente trabalho, Barbosa et al. (2009) observaram que, além da $\mathrm{CC}$, o peso dos animais também foi relacionado a variações em função do balanço energético. Cabras leiteiras com $\mathrm{CC}$ baixa e intermediária não diferiram entre si na sétima e oitava semanas de lactação, o que sugere condições semelhantes para esses animais a partir deste período, provavelmente em decorrência do aumento no consumo de MS das cabras mais magras durante a fase inicial da lactação, embora não tenha sido observada diferença entre cabras magras e com $\mathrm{CC}$ intermediária. Os animais de $\mathrm{CC}$ alta começaram a ganhar peso mais precocemente, a partir da segunda semana, em comparação aos dos outros grupos, nos quais o ganho de peso ocorreu apenas na terceira semana.
Ekanaes et al. (2006) avaliaram, por meio de tomografia computadorizada, as reservas corporais de gordura de cabras norueguesas com produção de $2,0 \mathrm{~kg} / \mathrm{cab} / \mathrm{dia}$, criadas em pastagens nativas durante toda a lactação e recebendo suplementação variando de 0,4 a $0,9 \mathrm{~kg} / \mathrm{cab}$. $/ \mathrm{dia}$, dependendo da qualidade da forragem. Esses autores observaram uma redução das reservas de gordura à medida que a lactação seguia. Aos 74 dias, quando houve a maior redução na produção de leite, as cabras já haviam mobilizado $53 \%$ da gordura que apresentavam no momento do parto, o que representou $67,5 \mathrm{~g}$ de tecido adiposo por dia. Comparativamente ao presente experimento, pressupõe-se que, até o fim do experimento, os animais ainda estariam mobilizando suas reservas corporais, quando comparado com o estudo anterior, mas estes já se encontravam em BEP em razão da baixa produção de leite estabelecida geneticamente.

A produção leiteira não foi um fator relevante para interferir na mobilização de reservas corporais de cabras leiterias de diferentes $\mathrm{CC}$ ao parto, com valores de $3,41 \pm 0,2 ; 3,44 \pm 0,2$ e $2,68 \pm 0,1 \mathrm{~kg}$ de leite/dia, respectivamente, para os grupos com baixa $\mathrm{CC}$, média $\mathrm{CC}$ e alta $\mathrm{CC}$, segundo Barbosa et al. (2009). Nesse sentido, a mobilização de reservas corporais para caprinos de média produção de leite parece não ser um fator influenciável no BEN, o que corrobora com a mobilização das reservas corporais neste período, que resulta em redução da $\mathrm{CC}$ e do peso corporal, sendo necessário para satisfazer as exigências de energia e proteína dos animais (Rodrigues et al., 2006).

Ao final da gestação, a CC ideal para se minimizarem os efeitos deletérios do BEN sobre a reprodução de caprinos não está estabelecida claramente, entretanto valores em torno de 3,5 parecem ser o ideal. Morand-Fehr et al. (1992), citados por Smith and Sherman (1994), no sistema de criação intensivo francês, mencionam que o escore final de $\mathrm{CC}$ de cabras leiteiras deve ser entre 2,75 e 3,5 ao parto. Entretanto, para cabras leiteiras confinadas em regiões tropicais no sudeste do Brasil, não se sabe qual a melhor $\mathrm{CC}$ ao parto e nos vários estágios de lactação (Rodrigues et al., 2006), necessitando de maiores estudos relacionando à $\mathrm{CC}$ ao parto e à atividade reprodutiva destas. 


\section{Influência da condição...}

Poucos trabalhos avaliaram os efeitos da $\mathrm{CC}$ ao parto e, consequentemente, do BEN em caprinos, sobre o retorno à atividade ovariana. Em cabras das raças Saanen e Anglo-Nubiana, criadas no nordeste do Brasil, foram verificadas uma duração média do anestro pós-parto de $95,26 \pm 11,80$ e $78,93 \pm 7,61$ dias, respectivamente
(Freitas et al., 2004). Além disso, Maia e Costa (1998), ao trabalharem com a raça Canindé, detectaram um período de 46 $\pm 3,4$ dias de anestro pós-parto e Andrioli, Simplício e Machado (1992) observaram anestro de 52.3 $\pm 3,9$ dias, em fêmeas sem padrão de raça definida (SPRD), paridas na estação seca.
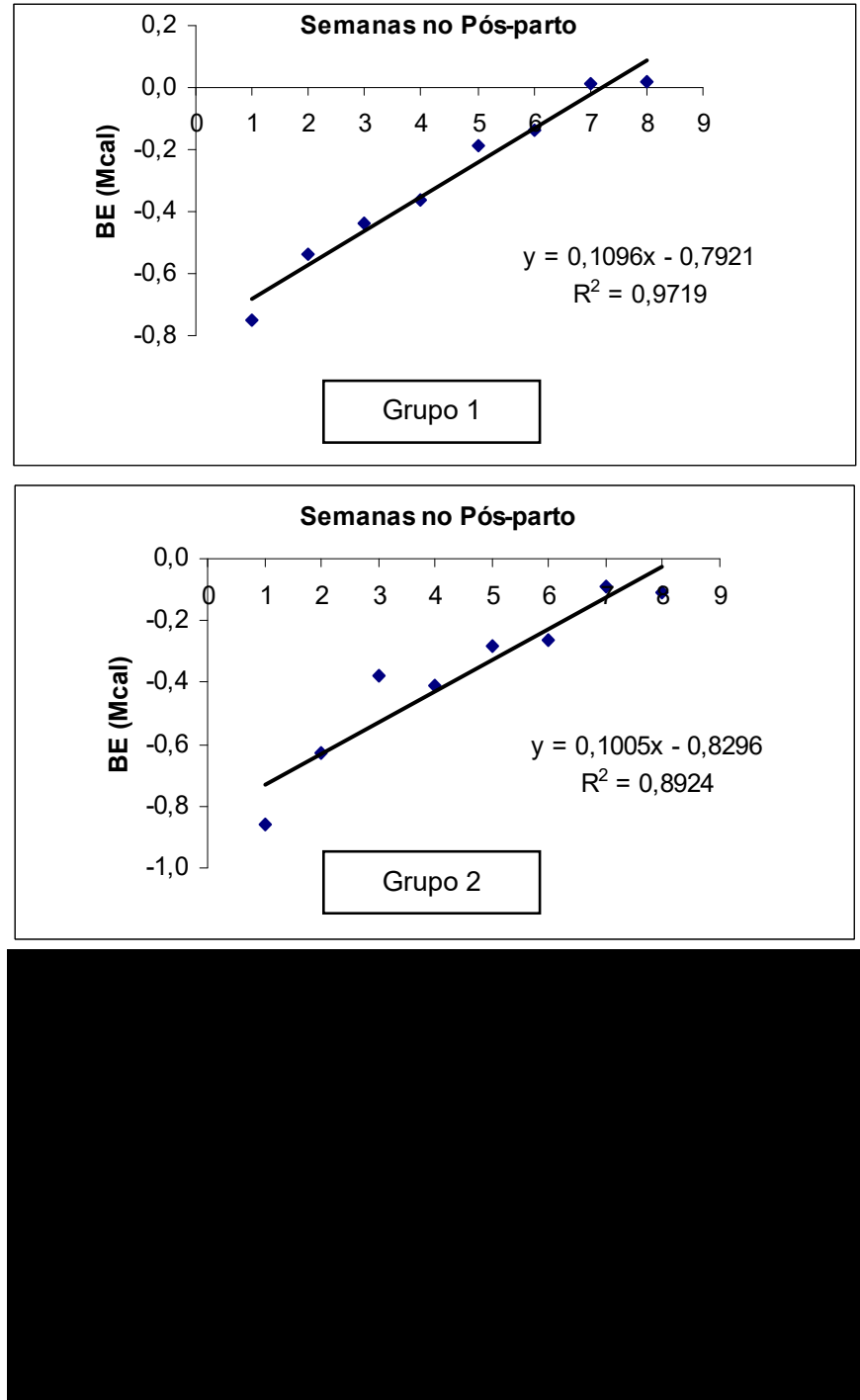

Figura 1. Balanço energético de cabras com baixa condição corporal, com moderada condição corporal e com alta condição corporal, em função do período pós-parto.

Em vacas leiteiras, a literatura indica que a $\mathrm{CC}$ parece ser programada geneticamente após o parto. Tal mecanismo é explicado pelo impulso biológico para mobilizar a reserva corporal em função da produção de leite (Garnsworthy, 2007). Caso o escore esteja acima deste alvo, o consumo de alimento é reduzido e perde-se CC; se o escore estiver abaixo deste alvo, o consumo de alimento aumenta e a vaca ganha $\mathrm{CC}$. Correlacionada com este fator a perda de 0,5 unidade de CC, observa-se uma diminuição da taxa de concepção em 10\% (Butler, 2005). Considerando isso, cabras de média produção parecem não mobilizar grandes quantidades de 
gordura para suprir suas exigências de energia para a produção de leite, especialmente quando estas recebem dietas de alta qualidade e alta concentração de energia. Em outras condições em que a dieta seja um fator limitante na fase de pós-parto para suprir a deficiência de energia dos animais, efeitos deletérios na reprodução poderiam ser observados.

Ao analisarem-se todas essas variáveis supracitadas: consumo, peso e produção de leite, pode-se entender de uma maneira mais clara o que aconteceu com o BEN dos animais do presente experimento. Assim, conjectura-se que, apesar de não haver diferenças no BEN entre os três grupos distintos de $\mathrm{CC}$ ao parto, pode-se estabelecer que as reservas de lipídeos são mobilizadas igualmente, pois o consumo não foi alterado, embora se tenha observado que houve uma mudança do peso dos animais, a qual se refletiu no balanço energético positivo (BEP) em fases diferentes do pós-parto.

Em associação ao CMS pós-parto, diversos autores relatam a influência da $\mathrm{CC}$ ao parto sobre os riscos de comprometimento das funções produtivas relacionadas à involução uterina lenta, ao anestro prolongado, ao ciclo estral curto e ao aumento do intervalo entre partos.

Em relação à exibição de estro pós-parto, apenas $4,4 \%$ (3/68) dos animais apresentaram estro detectável, sendo um do grupo de cabras com baixa $\mathrm{CC}$ e dois do grupo de cabras com moderada CC. Conforme observado por Zarazaga et al. (2015) a CC dos animais pode ter influenciado o número de folículos presentes no ovário antes da ovulação. Contrariamente, MezaHerrera et al. (2008) descreveram que o número de folículos pré-ovulatórios $\geq 5 \mathrm{~mm}$ não diferiu em cabras com alto $(3,2)$ ou baixo $(2,1)$ escores de CC. Chemineau et al. (2003) relatam que, em cabras após o parto, a ovulação é precedida da não manifestação de estro, em cabras da raça Crioula na terceira semana pós-parto. Nas condições de realização do presente experimento, embora não se tenha realizado ultrassonografia ovariana, pressupõe-se que o atraso na manifestação de estro se deva a outros fatores, como o fator social. Nas condições do presente experimento, os animais foram mantidos em baias individuais, e a observação de estro foi feita utilizando-se um macho introduzido na baia. Se as cabras fossem levadas ao macho ou agrupadas, provavelmente esse comportamento tivesse sido diferente, uma vez que o agrupamento de animais estimula a manifestação de estro.

Em ovelhas e cabras no pós-parto, as primeiras ovulações podem ser caracterizadas por um baixo pico de estrógeno, quando comparadas com animais não lactantes, com um menor pico de LH e, consequentemente, com baixas produções de progesterona pelo corpo lúteo, que apresenta um curto período de vida. Sugere-se como causa a influência luteolítica da involução uterina, devido ao aumento prolongado da liberação de $\mathrm{PGF}_{2} \alpha$ e à incompleta restauração da liberação de LH, ocorrendo insuficiente crescimento e maturação folicular (Gordon, 1997).

Leroy et al. (2006) afirmam que a energia necessária para o desenvolvimento, a maturação e a ovulação do folículo, para formar um corpo lúteo e para manter a fase inicial da gestação, é desprezível, se comparada à demanda de energia para iniciar e manter a lactação. Assim, é razoável supor que a poluição metabólica causada pelos produtos de degradação desse intenso tráfego de energia do trato digestório e de reservas corporais para o úbere seja responsável pela deterioração das funções reprodutivas em animais de alta produção, e não a falta de energia em si.

Dessa forma, a diferença encontrada no presente estudo de duas semanas para a mudança do $\mathrm{BE}$ para cabras com baixa $\mathrm{CC}$ e com alta $\mathrm{CC}$ pode apresentar importância fisiológica suficiente para interferir no restabelecimento das funções reprodutivas no pós-parto.

Diversos estudos foram reportados sobre o efeito do BEN e a menor taxa de concepção de vacas leiteiras de alta produção. Entretanto, para caprinos, apenas a derivação deste ocorrido tem sido levantada como hipótese, e, segundo o modelo de Britt (1992), que explica o efeito do BEN sobre a qualidade do oócito e do embrião gerado devido às condições adversas dessa fase de pós-parto, pode-se conjecturar que tal efeito acontece em cabras leiteiras. Além da menor qualidade do oócito e do embrião formados nessa fase de pós-parto, ocorre comprometimento à produção de progesterona pelo corpo lúteo resultante de um folículo de baixa qualidade 
(Britt, 1992). Segundo Butler (2003), o BEN tem grande associação com a frequência dos pulsos de LH e com os baixos níveis de glicose, insulina e IGF-1. Em conjunto, estes fatores limitam a produção de estrógeno pelo folículo dominante, diminuem a qualidade do oócito e a capacidade de desenvolvimento embrionário, além de reduzirem a concentração plasmática de progesterona.

Há evidência substancial de que a restrição alimentar e, consequentemente, a perda de $\mathrm{CC}$ e de peso também se inter-relacionam. Ferreira et al. (1990) mostraram que a perda de peso de $1 \mathrm{~kg} /$ dia influenciou a atividade ovariana em vacas mestiças ciclando, da mesma maneira que a realimentação e o ganho de CC.

Apesar disso, não houve diferença entre os grupos de diferentes $\mathrm{CC}$ quanto à concentração plasmática de progesterona $(\mathrm{P}>0,05)$, com apresentação de perfis semelhantes entre eles (Fig. 2).

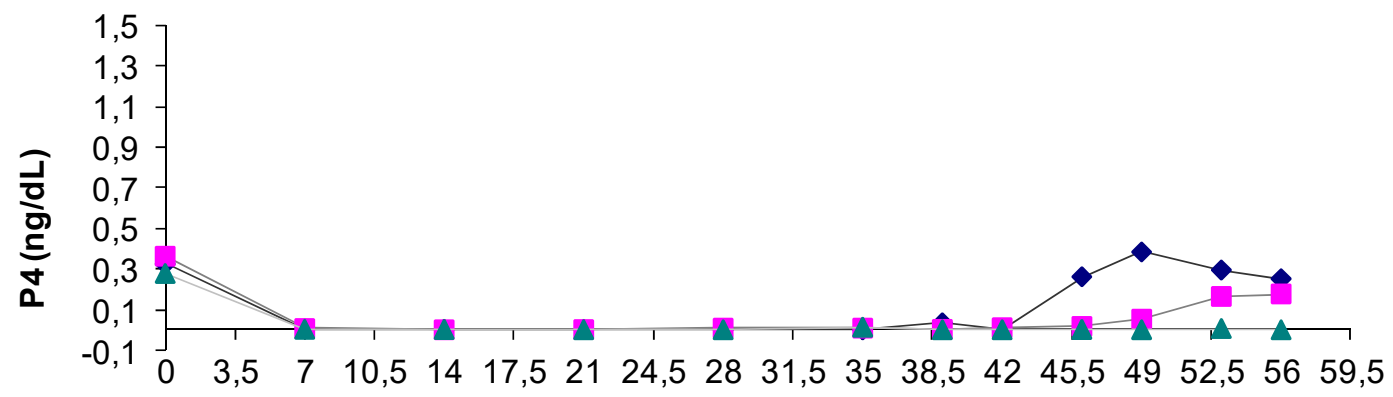

Dias no Pós-parto

$\longrightarrow$ Tratamento $1 \longrightarrow$ Tratamento $2 \backsim$ Tratamento 3

Figura 2. Concentração plasmática de progesterona de cabras com baixa condição corporal, com moderada condição corporal e com alta condição corporal em função do período pós-parto.

As concentrações plasmáticas de progesterona foram detectadas durante o parto, com concentração média de $0,32 \mathrm{ng} / \mathrm{dL}$, passando para concentrações não detectáveis durante as primeiras semanas de lactação e só voltando a serem detectáveis a partir da sexta semana de lactação. Khan e Ludri (2002) trabalharam com perfil hormonal de cabras no período do periparto e também mencionaram que as concentrações plasmáticas de progesterona declinaram entre o dia 20 pré-parto e o dia do parto, apresentando concentrações basais depois deste.

Apenas 5,9\% (4/68) dos animais apresentaram concentração plasmática de progesterona maior ou igual a $1 \mathrm{ng} / \mathrm{dL}$ durante as oito primeiras semanas de lactação, o que caracteriza função luteal (Fig. 2), sendo dois animais pertencentes ao grupo de cabras que pariram com baixa $\mathrm{CC}$ e dois ao grupo com moderada $\mathrm{CC}$; nenhum animal do grupo de cabras com alta $\mathrm{CC}$ apresentou ovulação durante o período experimental. A duração média do intervalo do parto ao primeiro estro foi de 46 dias. Esse valor difere dos reportados anteriormente na literatura referente ao anestro pós-parto em caprinos, entretanto nenhum dos dados citados anteriormente se assemelha às condições de alimentação, à localização e à CC dos animais do presente experimento.

\section{CONCLUSÕES}

Conclui-se que cabras leiteiras de média produção, entre 2,5 e 3,0kg de leite/dia, mesmo recebendo dieta com altos níveis de energia no pós-parto, entram em BE negativo, independentemente da condição corporal apresentada por elas ao parto, influenciando negativamente no reinício da atividade ovariana no pós-parto. 


\section{REFERÊNCIAS}

ADAMS, R.; COMERFORD, J.W.; FORD, S.A.; GRAVES, R.E. et al. Dairy reference manual: dairy nutrition. 3.ed. Ithaca: Northeast Regional Agricultural Engineering Service, 1995. p.129.

ANDRIOLI, A.; SIMPLICIO, A. A.; MACHADO, R. Influência da época da parição no comportamento reprodutivo pós-parto de cabras sem raça definida. Pesqui. Agropecu. Bras., v.27, p.65-72, 1992.

BARBOSA, L.P.; RODRIGUES, M.T.; GUIMARÃES, J.D. et al. Condição corporal e desempenho produtivo de cabras Alpinas no início da lactação. Rev. Bras. Zootec., v.38, p.2137-2143, 2009.

BRITT, J.H. Influence of nutrition and weight loss on reproduction and early embryonic death. In: WORLD BUIATRICS CONGRESS, 17., 1992, Saint Paul. Proceeding... Saint Paul: WAB, 1992. p.143-149.

BUTLER, W.R. Nutrition, negative energy balance and fertility in the postpartum dairy cows. Cattle Pract., v.13, p.13-18, 2005.

CHEMINEAU, P.; BARIL, G.; COGNIE, Y. et al. Training manual on artificial insemination in sheep and goats. Rome: FAO, 1993. v.83, p 258.

EKANAES, M.; KOLSTAD, K.; VOLDEN, H.; HOVE, K. Changes on body reserves and milk quality throughout lactation in dairy goats. Small Ruminant Res., v.63, p.1-11, 2006.

FERREIRA, A.M. Efeito da amamentação e do nível nutricional na atividade ovariana de vacas mestiças leiteiras. 1990. 134f. Tese (Doutorado em Zootecnia) - Universidade Federal de Viçosa, Viçosa, MG.

FREITAS, V.J.F.; RONDINA, D.; NOGUEIRA, D.M.; SIMPLÍCIO, A.A. Post-partum anoestrus in anglo-nubian and saanen goats raised in semiarid of north-eastern Brazil. Livest. Prod. Sci., v.90, p.219-226, 2004.

GARNSWORTHY, P. Manipulação do balanço energético e implicações na fertilizade. In: NOVOS ENFOQUES NA PRODUÇÃO E REPRODUÇÃO DE BOVINOS, 11., 2007, Uberlândia. Anais... Uberlândia: CONAPEC, 2007. p.12-21.
GONZALES, A.; MURPHY, B.D.; ALBA, J.; MANNS, J.G. Endocrinology of the postpartum period in the pelibuey ewe. J Anim. Sci., v.64, p.1717-1724, 1987.

GORDON, I. Controlled reproduction in sheep \& goats. New York: CAB International, 1997. v.2. 450p.

HAMADEH, S.K.; ABI SAID, M.; TAMI, F.; BARBOUR, E.K. Weaning and the ram-effect on fertility; serum luteinizing hormone and prolactin levels in spring rebreeding of postpartum Awassi ewes. Small Ruminant Res., v.41, p.91-194, 2001.

KHAN, J.R.; LUDRI, R.S. Hormonal profiles during periparturient period in single and twin fetus bearing goats. Aus. J. Anim. Sci., v.15, p.346-351, 2002.

LAGO, E.P.; PIRES, A.V.; SUSIN, I. et al. Efeito da condição corporal ao parto sobre alguns parâmetros do metabolismo energético, produção de leite e incidência de doenças no pósparto de vacas leiteiras. Rev. Bras. Zootec., v.30, p.1544-1549, 2001.

LEROY, J.L.M.R.; VAN SOOM, A.; KRUIF, A.; OPSOMER, G. Modern research in the reduced fertility in high producing dairy cows: an innovative way of thinking. Vlaams Diergeneeskd Tijdschr., v.75, p.18-22, 2006.

MAIA, M.; COSTA, A.N. Estro e atividade ovulatória pós-parto em cabras Canindé associados ao manejo da amamentação. Rev. Bras. Reprod. Anim., v.22, p.35-43, 1998.

MBAYAHAGA, J.; MANDIKI, S.N.M.; BISTER, J.L.; PAQUAY, R. Body weight, oestrous and ovarian activitiy in local Burundian ewes and goats after parturition in the dry season. Anim. Reprod. Sci., v.51, p.289-300, 1998.

MEZA-HERRERA, C.A.; HALLFORD, D.M.; ORTIZ, J.A.; CUEVAS, R.A.; SANCHEZ, J.M.; SALINAS, H. et al. Body condition and protein supplementation positively affect periovulatory ovarian activity by non LH-mediated pathways in goats. Anim. Reprod. Sci., v.106, p.412-20, 2008.

MOE, P.W.; TYRRELL, H.F. Symposium: production efficiency in the high producing cow: efficiency of conversion of digested energy to milk. J. Dairy Sci., v.58, p.602-610, 1975. 
MORAND-FEHR, P.; HERVIEU, J. Apprécier l'éat corporel des chèvres: intérêt et méthod. Reussir Chevre, n.231, 1999.

PARR, R.A.; DAVIS, I.F.; MILES, M.A.; SQUIRES, T.J. Feed intake affects metabolic clearance rate of progesterone in sheep. Res. Vet. Sci., v.55, p.306-310, 1993.

PEDRON, O.; CHELI, F.; SENATORE, E. et al. Effect of body condition score at calving on performance, some blood parameters, and milk fatty acid composition in dairy cows. J. Dairy Sci., v.76, p.2528-2535, 1993.

RODRIGUES, C.A.F.; RODRIGUES, M.T.; BRANCO, R.H. et al. Influência da condição corporal e da concentração de energia nas dietas no periparto sobre o desempenho de cabras em lactação. Rev. Bras. Zootec., v.35, p.1560-1567, 2006.

RUKKWAMSUK, T.; KRUIP, T.A.M.; MEIJER, G.A.L.; WENSING, T. Hepatic fatty acid composition in periparturient dairy cows with fatty liver induced by intake of a high energy diet in the dry period. J. Dairy Sci., v.82, p.280-287, 1999.

SMITH, M.C.; SHERMAN, D.M. Goat medicine. Philadelphia:Lea \& Febiger, 620p, 1994.
THE NUTRITION of goats. Wallingford: CAB International, 1998. 118p. (AFRC. Technical Committee on Response to Nutritients. Report, 10).

VAN SOEST, P.J. Integrated feeding systems. In: Nutritional ecology of the ruminant. 2.ed. Ithaca: Cornell University Press, 1994. 476p.

VERNON, R.G. Lipid metabolism in the adipose tissue of ruminant animals. In: CHRISTIE, W.W. (Ed.). Lipid metabolism in ruminant animals. New York: Pergamon Press, 1981. p.279-362.

VAZQUEZ-AÑON, M.; BERTICS, S.; LUCK, M.; et al. Peripartum liver triglyceride and plasma metabolites in dairy cows. J. Dairy Sci., v.77, p.1521-1528, 1994.

WALTERS, A.H.; BAILEY, T.L.; PEARSON, R.E.; GWAZDAUSKAS, F.C. Parity-related changes in bovine follicle and oocyte populations, oocyte quality, and hormones to 90 Days postpartum. J. Dairy Sci., v.85, p.824-832, 2002.

ZARAZAGA, L.A.; GUZMÁN, J.L.; DOMÍNGUEZ, C. et al. Effect of plane of nutrition on seasonality of reproduction in Spanish payoya goats. Anim. Reprod. Sci., v.87, p.253-267, 2005. 